Aus knapp 60 eingereichten Arbeiten wurden anlässlich des Nationalen Symposiums für Qualitätsmanagement im Gesundheitswesen am 15. Mai 2012 vier Projekte mit dem Swiss Quality Award 2012 ausgezeichnet. Die drei Trägerorganisationen IEFM, SQMH und die FMH freuen sich, in diesem Artikel die Gewinnerprojekte der Kategorien «Management» und «Technologie» vorzustellen. Die Siegerprojekte der Kategorien « $\mathrm{Pa}$ tientensicherheit» und «Empowerment» wurden in der letzten Ausgabe (24/2012) der Schweizerischen Ärztezeitung publiziert.

\title{
Swiss Quality Award 2012 - die Gewinner (Teil 2)
}

\section{Gewinner Kategorie Management}

\section{Volker Stange, Mara Völlmin, Willi Bäckert, \\ Remo Baumann, Udo Hartl, Mareile Endhardt, \\ Thierry Ettlin \\ * Der Begriff «Patientenmana- ger» umfasst immer die weibliche und männliche Form.}

\section{Korrespondenz:}

Mara Völlmin

dipl. Logopädin

Leiterin Patientenmanagement

Reha Rheinfelden

Salinenstrasse 98

CH-4310 Rheinfelden

Tel. 0618365301

m.voellmin[at]reha-rhf.ch

\section{Das Rheinfelder Patientenmanagement- Modell}

Im modernen Gesundheitswesen ist es erforderlich, sich unter Fachpersonen zu vernetzen, Prozesse abzubilden, den Personaleinsatz zu ökonomisieren und den Aufenthalt von Patienten optimal zu gestalten.

Die Reha Rheinfelden hat aus diesen Gründen das «Rheinfelder Patientenmanagement-Modell» geschaffen. Der Patientenmanager* begleitet den Patienten durch den ganzen Rehabilitationsprozess.

Ein kompetentes Projektteam hat das Rheinfelder Patientenmanagement-Modell entwickelt. Es legte besonderen Wert darauf, die Zielsetzung sowie die Aufgaben und Anforderungen klar zu erarbeiten, Kompetenzen und Verantwortlichkeiten zu klären, ferner das Schulungskonzept und mögliche Problemfelder zu definieren.

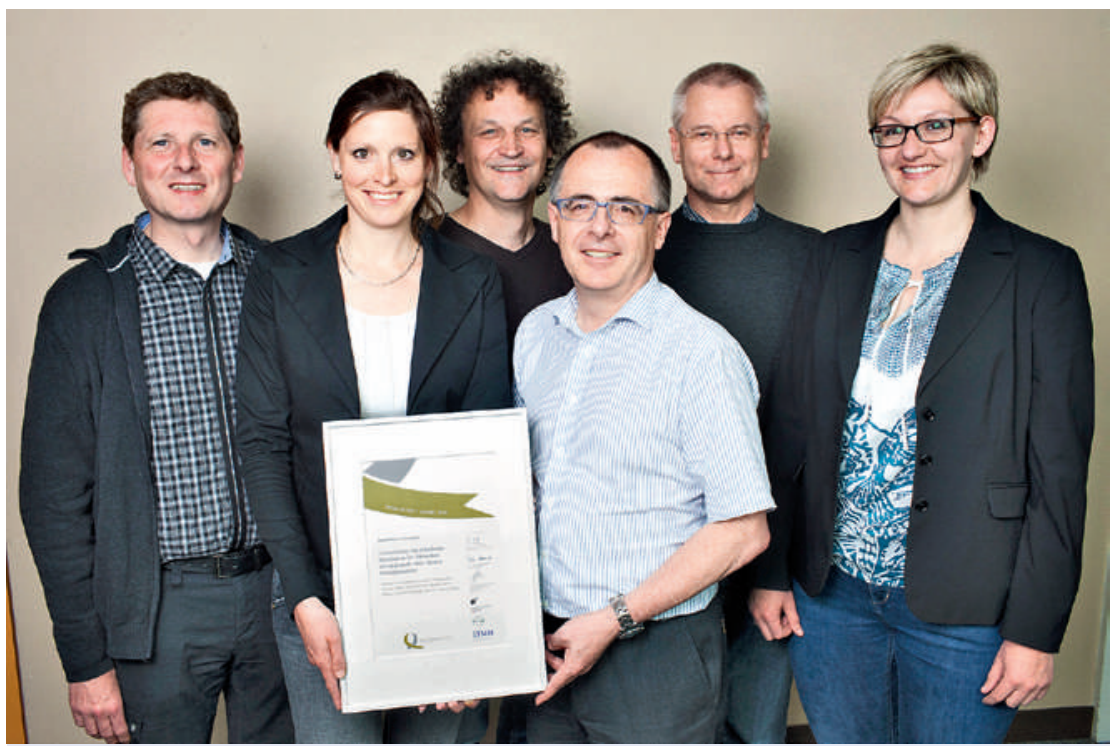

Gewinnerteam der Kategorie «Management» des Swiss Quality Awards 2012.

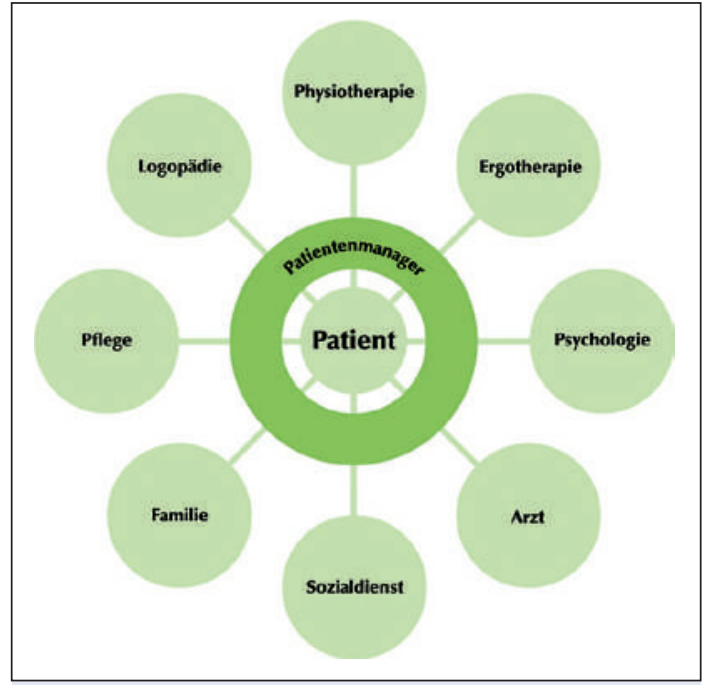

Der Patientenmanager ist Bindeglied und Mediator im Reha-Team.

Seit dem 1. September 2011 sind in der Reha Rheinfelden zehn Patientenmanager auf zehn ärztlichen Abteilungen tätig. Diese werden nach strengen Kriterien bezüglich der fachlichen Kompetenzen, der Erfahrung in der Rehabilitation, des organisatorischen Talentes und der Kommunikationskompetenz intern aus den therapeutischen und pflegerischen Berufen rekrutiert.

Als persönliches Bindeglied zwischen dem Patienten, den verschiedenen Berufsgruppen in der Medizin und der Administration sind unsere Patientenmanager im Netzwerk Rehabilitationsklinik tätig. Als feste Ansprechpartner begleiten sie den Patienten durch seinen Rehabilitationsaufenthalt, koordinieren Abläufe und gewährleisten den interdisziplinären Informationsaustausch und eine bestmögliche ambulante Weiterversorgung nach dem Austritt. Am Eintrittstag nimmt unser Patientenmanager persönlichen Kontakt mit dem Patienten auf, heisst ihn 
willkommen, führt ein Vorgespräch und erstellt in Zusammenarbeit mit dem Arzt eine individuelle Therapieverordnung. Er organisiert nötige Hilfsmittel und beantwortet erste Fragen.

Während des Aufenthaltes stellt der Patientenmanager in Zusammenarbeit mit dem Behandlungsteam sicher, dass der Patient ein optimales Therapieprogramm erhält. Für die Dokumentation des Rehabilitationsverlaufes wird von allen Beteiligten ein elektronisch geführtes Dokument, das Interdisziplinäre Verlaufsprotokoll (IVP), genutzt. Der Patientenmanager hat die Verantwortung für die korrekte

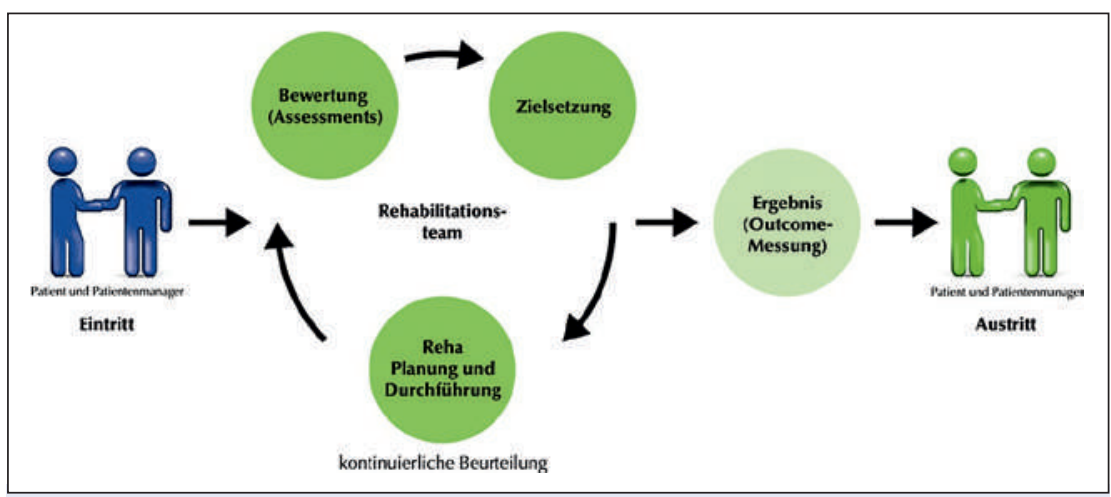

Patient und Patientenmanager im Rehabilitationsprozess.

\section{Gewinner Kategorie Technologie}

\section{Andreas Meer, Corina Costea, Adrian Wirthner}

Korrespondenz:

Dr. med. A. Meer, MHIM

Facharzt Allgemeine Medizin

FMH

in 4 medicine AG

Pavillonweg 3

CH-3012 Bern

Tel. 0313701330

a.meer[at]in4medicine.ch

www.in4medicine.ch

\section{Onlinepraxis}

\section{Vom Projekt zum Produkt}

Das Projekt Onlinepraxis wurde vor vier Jahren durch das Ärztenetzwerk mediX und die Firma in4medicine ins Leben gerufen [1]. Das Projekt hatte zum Ziel:

- eine datenschutzkonforme Internetplattform zu entwickeln, welche es den Haus- und Spezialärzten ermöglicht, ihre Patienten - ergänzend zu den Konsultationen im Sprechzimmer - auch online zu betreuen.

- die Behandlungsqualität hinsichtlich Patientenorientierung, Sicherheit, Effizienz, Effektivität und Verfügbarkeit zu verbessern [2].

- die Webanwendung so zu entwickeln, dass sich Daten mit der elektronischen Krankengeschichte austauschen lassen und der Umgang mit der Onlinepraxis möglichst einfach und benutzerfreundlich ist.

Im Frühjahr 2010 begann die Softwareentwicklung. Im Herbst 2011 starteten die Ärzte der Praxis Bubenberg in Bern mit der Onlinebetreuung ihrer Patienten. Im Januar 2012 nahm die mediX Gruppenpraxis
Führung des IVP und stellt auf den Kaderarztvisiten den Informationsaustausch zwischen Ärzten und anderen Berufsgruppen her. Wöchentlich führt der Patientenmanager Therapievisiten beim Patienten durch, um Fragen und Wünschen nachzugehen sowie das Therapieprogramm zu kontrollieren und individuell anzupassen.

Durch das «Patientenmanagement» gewinnt die Kommunikation zwischen Arzt, Patient und Patientenmanager in emotionaler und zwischenmenschlicher Hinsicht, der Kontakt wird persönlicher, der Mensch hinter der Rolle sichtbarer und authentischer; das hat positive Auswirkungen auf den Rehabilitationserfolg.

Die Patientenmanager kommen aus unterschiedlichen medizinischen Berufen, deshalb ist eine gute anfängliche und kontinuierliche Schulung von grosser Bedeutung. Wichtig ist der Austausch unter den Patientenmanagern, in dem der Einzelne seine Stärken und sein Wissen anderen zur Verfügung stellt. Die Tätigkeit in den Zwischenzonen von unterschiedlichen Kompetenz- und Verantwortungsbereichen geht nicht ohne Reibungen einher, daher sind Fähigkeiten im Konfliktmanagement notwendig.

Das Patientenmanagement hat sich in unserem Haus gut etabliert und wird sich dynamisch in die Zukunft hinein entwickeln.

Die Rückmeldungen sind positiv.

in Zürich den Betrieb der Onlinepraxis auf. Im Verlaufe des Jahres 2012 wird der Kundenkreis auf mehrere Praxen in der Schweiz erweitert.

\section{Die Onlinepraxis}

Gegenwärtig bietet die Onlinepraxis die folgenden Elemente der Patientenbetreuung:

- Online-Konsultation: virtuelle Konsultationen zwischen Arzt und Patient.

- Online-Resultat: Mitteilung ärztlich validierter und bei Bedarf kommentierter Untersuchungsresultate direkt aus der elektronischen Krankengeschichte.

- Online-Pass: die Patienten haben jederzeit und überall Einsicht in die wichtigsten, durch ihren Arzt validierten und aktualisierten Daten der elektronischen Krankengeschichte (z. B. Medikamente, Allergien).

- Online-Administration: administrative Anfragen an die Medizinische Praxisassistentinnen (z.B. Terminanfrage, Rezepte).

\section{Erste Resultate}

Die ersten Erhebungen zeigen folgende Ergebnisse in Hinblick auf die in den Zielen des Projektes 


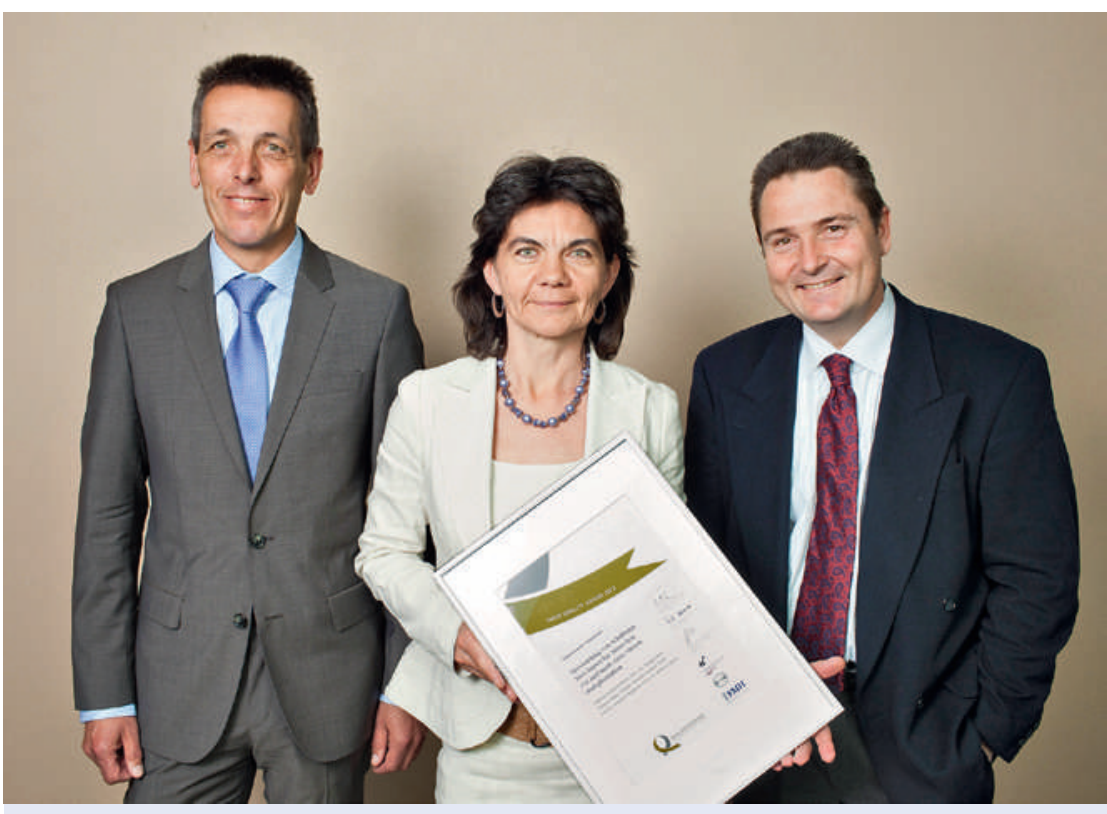

Gewinnerteam der Kategorie «Technologie» des Swiss Quality Awards 2012, A. Wirthner, C. Costea, A. Meer.
- Die Patienten geben spontan Rückmeldungen zum Behandlungsverlauf. Dies verbessert die Kontinuität der Betreuung.

\section{Schlussfolgerungen und Ausblick}

Mit der Onlinepraxis wurde in den Schweizer Arztpraxen eine Technologie eingeführt, welche den Praxisalltag und die Art und Weise, wie Patienten hausund spezialärztlich betreut werden, verändert. Das Projekt Onlinepraxis ist ein soziotechnologisches Projekt. Es zeigt auf, wie eine Technologie zu neuen Formen der Patientenbetreuung und zu einer verbesserten medizinischen Behandlungsqualität führen kann. mediX und in4medicine sind bestrebt, den Veränderungsprozess wissenschaftlich zu evaluieren und die Erkenntnisse in die laufenden Debatten zur Qualitäts- und eHealth-Strategie des Bundes im Schweizerischen Gesundheitswesen einzubringen.

\section{Literatur}

1 www.onlinepraxis.ch

2 Institute of Medicine, Committee on Quality of Health Care in America. Crossing the quality chasm - A New Health System for the 21st Century. Washington, D.C.: National Academy Press; 2001.

aufgeführten Dimensionen der Behandlungsqualität:

Zur Patientenorientierung und Sicherheit

- Die Online Konsultationen werden seitens der Patienten ganz selbstverständlich als integrierter Bestandteil einer hausärztlichen Betreuung in Anspruch genommen und sehr geschätzt.

- Der Konsultationsanlass steht meist im Kontext einer andauernden Erkrankung und einer etablierten Patienten-Arzt-Beziehung.

- Der Informationsaustausch ist schriftlich, die Kommunikation wird verbindlicher.

- Die Betreuung über die Onlinepraxis ist datenschutzkonform und respektiert die Privatsphäre der Patienten.

\section{Zur Effizienz, Effektivität und Verfügbarkeit}

- Vor allem Folgekonsultationen und die Mitteilung beziehungsweise der Kommentar von Untersuchungsresultaten können effizient online erfolgen.

- Die Online-Konsultationen ersetzen telefonische Konsultationen; die Sprechstunde wird weniger gestört.

- Die Konsultationszeiten sind kurz. Die Konsultationen können praktisch ausschliesslich online abgeschlossen werden.

\section{Swiss Quality Award - Innovations in Healthcare}

Der Swiss Quality Award wird in den vier Kategorien Management, Patientensicherheit, Technologie und Empowerment verliehen. Getragen wird der Preis von drei Organisationen: der nagement im Gesundheitswesen (SQMH), dem Institut für Evaluative Forschung in der Medizin (IEFM) der Universität Bern sowie der Verbindung der Schweizer Ärztinnen und Ärzte (FMH). Alle Informationen zum Swiss Quality Award sowie eine Onlineplattform mit den Projektpostern der Gewinner und weiteren Projekten befinden sich unter www.swissqualityaward.ch.

\section{Ausschreibung 2013}

Nach der Preisverleihung ist vor der Preisverleihung! Der Swiss Quality Award wird auch 2013 erneut verliehen. Nebst neuen Projekten sind auch Projekte willkommen, die sich schon mal um den Swiss Quality Award beworben haben. Bedingung ist jedoch, dass sich ein Projekt deutlich weiterentwickelt hat und diese Entwicklung auch dokumentiert wird. Die Preisausschreibung ist für den Herbst 2012 geplant. Schweizerischen Gesellschaft für Qualitätsma- 